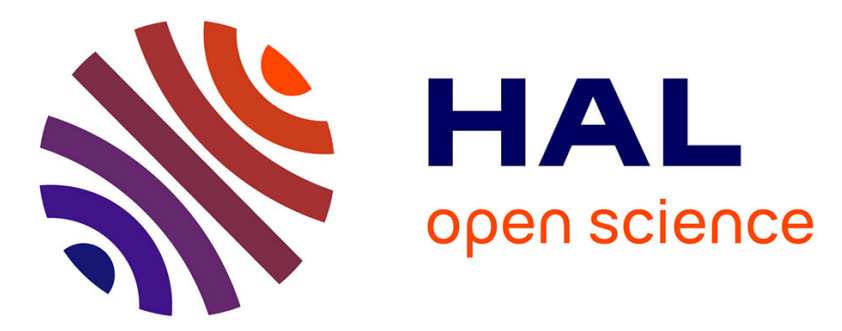

\title{
Acoustic radiation force on small spheres due to transient acoustic fields
}

Qing Wang, Antoine Riaud, Jia Zhou, Zhixiong Gong, Michael Baudoin

\section{To cite this version:}

Qing Wang, Antoine Riaud, Jia Zhou, Zhixiong Gong, Michael Baudoin. Acoustic radiation force on small spheres due to transient acoustic fields. Physical Review Applied, 2021, 15 (4), pp.044034. 10.1103/physrevapplied.15.044034 . hal-03347649

\section{HAL Id: hal-03347649 \\ https://hal.science/hal-03347649}

Submitted on 17 Sep 2021

HAL is a multi-disciplinary open access archive for the deposit and dissemination of scientific research documents, whether they are published or not. The documents may come from teaching and research institutions in France or abroad, or from public or private research centers.
L'archive ouverte pluridisciplinaire HAL, est destinée au dépôt et à la diffusion de documents scientifiques de niveau recherche, publiés ou non, émanant des établissements d'enseignement et de recherche français ou étrangers, des laboratoires publics ou privés. 


\title{
Acoustic Radiation Force on Small Spheres Due to Transient Acoustic Fields
}

\author{
Qing Wang, ${ }^{1}$ Antoine Riaud $\odot,{ }^{1, *}$ Jia Zhou $\odot,{ }^{1}$ Zhixiong Gong $\odot,{ }^{2}$ and Michael Baudoin $\odot^{2, \dagger}$ \\ ${ }^{1}$ State Key Laboratory of ASIC and System, School of Microelectronics, Fudan University, \\ Shanghai 200433, China \\ ${ }^{2}$ University of Lille, CNRS, Centrale Lille, University Polytechnique Hauts-de-France, UMR 8520-IEMN, \\ Lille F-59000, France
}

(Received 17 November 2020; revised 11 March 2021; accepted 30 March 2021; published 21 April 2021)

Acoustic radiation force is a net force experienced by an object under the action of an acoustic wave. Most theoretical models require the acoustic wave to be periodic, if not purely monofrequency, and are therefore irrelevant for the study of acoustic radiation force due to acoustic pulses. Here, we introduce the concept of finite-duration pulses, which is the most general condition to derive the acoustic radiation force. In the case of small spheres, we extend the Gor'kov formula to unsteady acoustic fields such as traveling pulses and interfering wave packets. In the latter case, our study suggests that the concept of acoustic contrast is also relevant to express the acoustic radiation force. For negative acoustic contrast particles, the acoustic trapping region narrows with shorter pulses, whereas positive contrast particles (such as biological cells) can fall in secondary traps when the pulse width deviates from an optimal value. This theoretical insight may help to improve the selectivity of pulsed acoustic tweezers.

DOI: 10.1103/PhysRevApplied.15.044034

\section{INTRODUCTION}

Acoustic radiation pressure is generally described as a steady force acting on surfaces exposed to acoustic waves. While radiation pressure has been extensively studied for almost two centuries, the steady nature of the force has been little discussed. Since the pioneering work of Rayleigh [1,2], radiation pressure has mainly been studied in the monofrequency regime and been defined as an exchange of momentum between a wave and a particle. Integrating the acoustic radiation pressure over an object's boundaries yields a net acoustic radiation force, which has been computed for incompressible spheres exposed to plane waves by King [3] and later on by taking the sphere compressibility into account by Yosioka and Kawasima [4]. In 1962, Gor'kov derived a formula for the acoustic radiation force of spheres much smaller than the acoustic wavelength subject to arbitrary acoustic fields [5]. Even though these calculations have been extended to arbitrary large spheres in complex acoustic fields [6-8], and to include viscous effects and even acoustothermal effects [9-13], the monofrequency dogma has remained essentially unchallenged.

Silva et al. have investigated the parametric oscillations of spheres of arbitrary size exposed to bichromatic and polychromatic acoustic waves in an inviscid fluid [14,15].

\footnotetext{
*antoine_riaud@fudan.edu.cn; http:/homepage.fudan.edu.cn/ariaud/

†'michael.baudoin@univ-lille.fr
}

They have shown that, unlike the monochromatic case, the amplitude of the parametric forcing depends on the nonlinearities in the fluid equation of state. A simplified expression of the acoustic radiation force was independently provided by Karlsen and Bruus for the average force experienced by small spheres exposed to polychromatic waves in a thermoviscous fluid [13]. In both cases, the polychromatic assumption refers to a discrete combination of modes, which is tantamount to periodic excitation signals. Although this allows the analysis of a combination of transducers [16,17], the periodic assumption makes it a priori irrelevant for continuous combinations of frequencies [18] and acoustic pulses [19].

While the traditional use of acoustic radiation force for acoustic levitation and acoustic tweezing allows extremely long actuation signals to be considered, and is therefore well described by existing theories, using a continuous range of frequencies offers a superior flexibility in device operation. This enables finely tuning the spatial geometry of an acoustic field, which is tantamount to adjusting the acoustic radiation force landscape. For instance, Kang et al. have demonstrated such a multifrequency device [18] for the fine positioning of particles. However, one of the most interesting prospects of pulses is to increase the acoustic trapping selectivity, that is the ability to trap a particle within many others by confining the acoustic field around this particle. Unlike the complex transducers [20,21] or transducer arrays [22-24] typically needed to achieve a high selectivity, Collins et al. have pioneered an alternative strategy using ultrashort acoustic pulses [19]. 
They assumed that, similarly to the monofrequency case, traveling acoustic pulses would generate much less acoustic force than standing acoustic pulses (obtained by the interference between two counterpropagating pulses). They demonstrated that an acoustic pulse width as short as 10 acoustic periods yields an acoustic radiation force, and that acoustic interference and trapping regions overlap. Even though the limited bandwidth of their transducers restricted the pulse shortness to at least 10 periods, it not only challenges the monofrequency assumption, but also suggests that further reducing the pulse width may enable even higher selectivity.

Furthermore, nonconventional acoustic generation methods such as percussions and photoacoustic effects $[25,26]$ can only generate brief impulsions with a broad frequency content. Starting from Longhorn's study of the effect of shock waves on small particles [27], the hydrodynamic community has also considered the forces generated by fast variations of pressure and velocity [28,29]. Yet, even the most recent refinement have overlooked the effect of the sphere compressibility and describe complex dynamics on timescales that cannot be resolved experimentally.

In this paper, we question the steady nature of the acoustic radiation force by considering acoustic wave packets of finite duration $\tau$. By finite duration, we require all the wave quantities $\tilde{x}$ to satisfy the condition $\tilde{x}(-\tau / 2, \mathbf{r})=$ $\tilde{x}(\tau / 2, \mathbf{r})$, where the origin of time is freely chosen. Such a wave packet is shown in Fig. $1(\mathrm{~b})$, where $\tilde{x}(-\tau / 2, \mathbf{r})=$ $\tilde{x}(\tau / 2, \mathbf{r})=0$. Note that the duration $\tau$ can be arbitrarily larger than the pulse width $\Theta$ (the time window containing most of the acoustic energy of the pulse).

This finite-duration condition is a considerably more relaxed requirement than periodic wave packets [where the equality $\tilde{x}(t-\tau / 2, \mathbf{r})=\tilde{x}(t+\tau / 2, \mathbf{r})$ must hold for all times $t$, and even more so for monofrequency waves. We also note that such packets allow the construction of periodic wave packets and monofrequency waves by juxtaposing several periods. While the conditions on the acoustic wave are relaxed, we now need to assume that the acoustic field experienced by the particle does not change much during the entire pulse duration. Since acoustic quantities vary over a wavelength, this condition is tantamount to assuming that the particle displacement $\Delta \ell$ is negligible compared to the shortest acoustic wavelength $\lambda$ of the excitation signal (corresponding to the highest frequency $f_{\max }$ in the pulse), not only during an acoustic period, but during the entire acoustic pulse duration, that is $\Delta \ell / \lambda \ll 1$. Similarly to previous theoretical studies on the acoustic radiation force, our calculations rely on the perturbation expansion to evaluate the force, which in turn restricts the particle migration speed $v_{p} \simeq \Delta \ell / \Theta \gtrsim$ $\Delta \ell / \tau$ to $v_{p} / c_{0} \lesssim \epsilon^{2}$ [13], with $\epsilon=p_{\max } / \rho_{0} c_{0}^{2}$ the acoustic Mach number, where $\rho_{0}, c_{0}$, and $p_{\max }$ are the fluid density at rest, the wave speed, and the peak wave-pressure
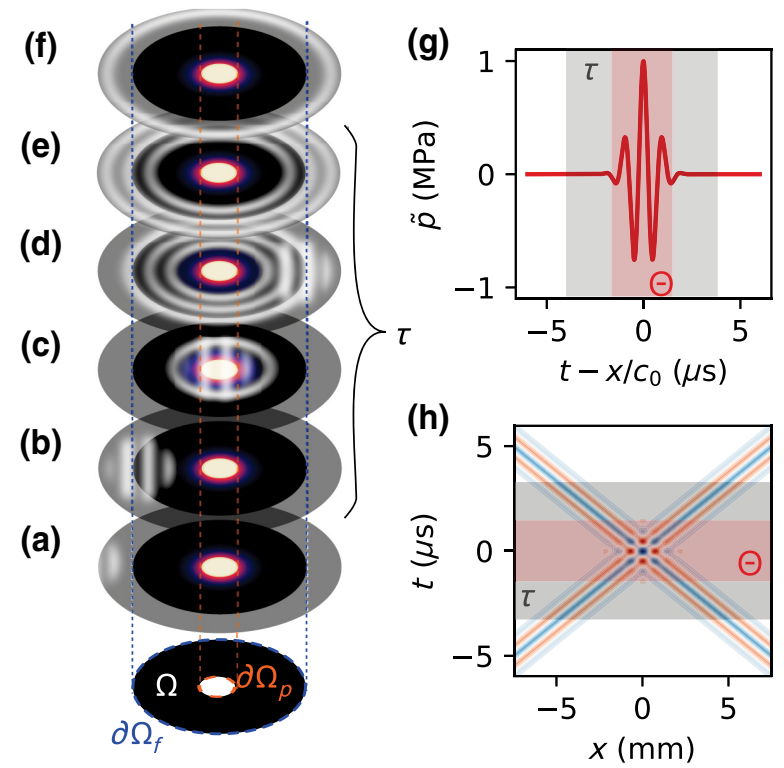

FIG. 1. Scattering and interference of wave packets. (a)-(f) Time lapse of the diffraction of an acoustic wave by a wave packet. A particle (white disk) surrounded by viscous and thermal boundary layers (blue and red) sits in a quiescent fluid. The domain $\Omega(t)$ extends from the particle surface $\partial \Omega_{p}(t)$ to a fixed boundary $\partial \Omega_{f}$ several acoustic wavelengths away, and encompasses both boundary layers. At (a),(b), a wave packet enters $\Omega$, then (c) is scattered by the particle and (c)-(e) generates a scattered field (shown as circular waves) that (f) eventually exits $\Omega$. Neglecting the particle motion, the state of $\Omega$ is identical in steps (a),(f), which allows defining $\tau$ as the duration between these two steps. (g) A Gaussian acoustic pulse. The gray region indicates the pulse duration $\tau$, which can be chosen arbitrarily larger than the pulse width $\Theta$ (in red). (h) Interference of two Gaussian pulses.

amplitude, respectively. This yields a coarse overestimate of this small-displacement condition:

$$
\frac{\Delta \ell}{\lambda} \simeq \Theta f_{\max } \epsilon^{2} \leq \tau f_{\max } \epsilon^{2} \ll 1
$$

The small $\epsilon \lesssim 0.01$ for most acoustofluidic applications suggests that the above inequalities hold as long as $\tau \lesssim$ 100 acoustic periods long. Assuming that the excitation signal bandwidth is bounded by $\left[f_{\min }, f_{\max }\right]$, this finite-pulse theory will be relevant for $f_{\min } \gg f_{\max } \epsilon^{2} \sim 10^{-4} f_{\max }$. More stringent conditions are foreseen when the particle can resonate with the incident field and emit the scattered field for a longer duration. This may occur for bubbles or particles with a size comparable to the acoustic wavelength. For periodic series of pulses, this has little consequences because the acoustic field variables will cycle over the pulse repetition period so that the acoustic radiation force of each pulse period can be evaluated by carefully including the steady-state scattered field due to this periodic 
excitation. For isolated or stochastic acoustic pulses however, the finite-duration condition would require $\tau$ large enough to allow the scattered field to decay to negligible levels before evaluating the acoustic radiation force.

This finite duration allows us to overlook the complex dynamics found by Longhorn and subsequent studies [14, 15,27-30], which are difficult to resolve experimentally at present [30,31], and to focus instead on the transformation of the particle from the initial state before wave-packet scattering and a final state after the scattering event. This is illustrated in Figs. 1(a)-1(f): the domain $\Omega$ contains a particle (white disc) with a thermoviscous boundary layer (in blue and red). An incident wave packet falls on the particle and generates a scattered field that eventually escapes $\Omega$ at step (f). The finite-duration condition requires that the state of $\Omega$ at step (f) is the same as in step (a).

After generalizing the expression of the acoustic radiation pressure tensor to finite-duration wave packets, we rederive the acoustic radiation force on small spheres while relaxing the monofrequency assumption. This will provide a generalized Gor'kov equation for acoustic wave packets of finite duration. Although our final expression could be derived from Karlsen et al.'s expression for periodic waves [13] using Parseval's theorem, and even for nonperiodic pulses using Plancherel theorem, (i) rederiving the equation provides a deeper physical meaning of its origin, especially regarding the scattering coefficients, and (ii) our derivation has a broader set of validity than what these theorems alone would warrant. In particular, using the Plancherel theorem requires integration of the time-domain function over $[-\infty,+\infty]$ and would therefore not ensure, for instance, that successive pulses would not interfere. After deriving this general expression, we then evaluate the acoustic radiation force of these packets in the simplified cases of traveling and standing waves, and use our analytical expression to optimize the selectivity of acoustic trapping by acoustic pulses.

\section{MODEL}

\section{A. Definition of the acoustic radiation force}

Using the summation of repeated indices, mass and momentum conservation in a Newtonian fluid of shear viscosity $\mu$ and bulk viscosity $\mu_{b}$ read

$$
\begin{aligned}
\partial_{t} \rho+\partial_{i} \rho v_{i} & =0 \\
\partial_{t} \rho v_{i}+\partial_{j} \rho v_{i} v_{j} & =\partial_{j}\left(-p \delta_{i j}+\Sigma_{i j}\right) \\
\Sigma_{i j} & =\mu\left(\partial_{j} v_{i}+\partial_{i} v_{j}\right)+\left(\frac{\mu}{3}+\mu_{b}\right) \delta_{i j} \partial_{j} v_{j}
\end{aligned}
$$

with $p, \rho$, and $\mathbf{v}$, the fluid pressure, density, and velocity, and $\delta_{i j}$ is the Kronecker $\delta$ function. These equations are complemented with the isentropic equation of state up to the second order in $\rho-\rho_{0}$ :

$$
p-p_{0}=c_{0}^{2}\left(\rho-\rho_{0}\right)+\frac{1}{2} \Gamma\left(\rho-\rho_{0}\right),
$$

with $\Gamma=(B / A)\left(c_{0}^{2} / \rho_{0}\right)$, where $B / A$ is the nonlinear parameter $[14,32]$.

A particle with boundaries located at $\partial \Omega_{p}(t)$ immersed in this fluid will experience a force $F_{i}^{p}=\oint_{\partial \Omega_{p}(t)} p \delta_{i j}-$ $\Sigma_{i j} d S_{j}^{p}[13,32]$. When the fluid motion is solely due to acoustic waves, the time average of this force is the acoustic radiation force. However, the particle position and shape are affected by the acoustic field, meaning that the integration boundary $\partial \Omega_{p}$ varies with time. This problem is addressed by setting a fixed boundary $\partial \Omega_{f}$ arbitrarily far away from the particle [32], and integrating Eq. (2b) over the volume $\Omega(t)$ delimited by this boundary and the particle moving surface. After using the Gauss integral theorem, we get

$$
\begin{gathered}
\int_{\Omega(t)} \partial_{t} \rho v_{i} d V+\int_{\partial \Omega_{f}}\left(\rho v_{i} v_{j}+p \delta_{i j}-\Sigma_{i j}\right) d S_{j} \\
-\int_{\partial \Omega_{p}(t)}\left(\rho v_{i} v_{j}+p \delta_{i j}-\Sigma_{i j}\right) d S_{j}^{p}=0
\end{gathered}
$$

where the negative sign of the particle boundary integral indicates that the surface unit vector is pointing towards the particle center.

Equation (4) is then combined with the Reynolds transport equation $\partial_{t} \int_{\Omega(t)} \rho v_{i} d V=\int_{\Omega(t)} \partial_{t} \rho v_{i} d V+$ $\int_{\partial \Omega_{f}} \rho v_{i} v_{j}^{b} d S_{j}+\int_{\partial \Omega_{p}(t)} \rho v_{i} v_{j}^{b} d S_{j}$, with $\mathbf{v}^{b}$ the displacement speed of the integral bounds. Noting that on the fixed boundary $\left(\partial \Omega_{f}\right), \mathbf{v}^{b}=\mathbf{0}$; and on the particle boundary $\left[\partial \Omega_{p}(t)\right], \mathbf{v}^{b}=\mathbf{v}$ by continuity, we can evaluate the force on the particle by knowing the acoustic field on a remote fixed boundary:

$$
\partial_{t} \int_{\Omega(t)} \rho v_{i} d V+\int_{\partial \Omega_{f}} \mathcal{B}_{i j}^{\text {tot }} d S_{j}=F_{i}^{p}
$$

with $\mathcal{B}_{i j}^{\text {tot }}=\rho v_{i} v_{j}+p \delta_{i j}-\Sigma_{i j}$ the flux of momentum through $\partial \Omega_{f}$.

The main caveat of working on a remote boundary is that it operates a momentum balance over the entire domain $\Omega$, such that acoustic momentum transfer from the wave to the fluid inside $\Omega$, and the steady flow generated by the acoustic wave outside this domain (acoustic streaming [33]) are included in the left-hand term [32]. It is customary to distinguish three contributions, namely the acoustic momentum that would have been transmitted to the fluid even in the absence of any particle (such as Eckart [34] and Rayleigh streaming [35]), the acoustic momentum exchanged with the particle by scattering, 
and the acoustic momentum exchanged with the particle by local steady viscous stress (acoustic microstreaming [36]). Except for very special cases such as heavy particles in viscous fluids [37], the viscoacoustic boundary layer is generally thinner than the particle size such that the microstreaming contribution to the acoustic radiation force is negligible [37]. Then, the acoustic radiation force is well approximated by considering only the inviscid contribution, as long as the thermoviscous effects on the scattering coefficients are well resolved in the vicinity of the particle $[12,13]$. Therefore, we neglect the contribution of microstreaming, which allows useful simplifications in the calculation of the acoustic radiation force. The contribution of Rayleigh and Eckart streaming may then be added as a drag force on the particle [32,38].

\section{B. Perturbation expansion in the far field}

In most experiments, the spatial scale of acoustic attenuation $\Lambda_{\mathrm{ac}}=\rho_{0} c_{0}{ }^{3} /\left[\mu\left(4 / 3+\mu_{b} / \mu\right) \omega^{2}\right]$ is many orders of magnitude larger than the scale of the viscous $\Lambda_{\text {visc }}=$ $\sqrt{2\left(\mu / \rho_{0} \omega\right)}$, and thermal $\Lambda_{\text {therm }}=\sqrt{2\left(D_{T} / \omega\right)}$ boundary layers (with $D_{T}$ the thermal diffusivity in the fluid), so that the effect of these layers on the acoustic radiation force can be resolved locally while the propagation of acoustic waves is well approximated by considering an inviscid and adiabatic fluid $[12,13]$. Therefore, we now omit the viscous effects in the fluid, and use the small acoustic Mach number $\epsilon$ to expand the evolution of the pressure, velocity, and density fields by perturbation of increasing orders in $\epsilon$. Zero-order quantities are denoted $x_{0}$, first-order $\tilde{x}$, and second-order $\bar{x}$ :

$$
\begin{aligned}
& \rho=\rho_{0}+\epsilon \tilde{\rho}+\epsilon^{2} \bar{\rho}, \\
& p=p_{0}+\epsilon \tilde{p}+\epsilon^{2} \bar{p}, \\
& v_{i}=0+\epsilon \tilde{v}_{i}+\epsilon^{2} \bar{v}_{i} .
\end{aligned}
$$

In order to get nontrivial values for the acoustic Mach number, we now solve each perturbation order independently. The order 0 in $\epsilon$ is the hydrostatic equilibrium:

$$
\begin{aligned}
& \partial_{t} \rho_{0}=0, \\
& \partial_{i} p_{0}=0,
\end{aligned}
$$

which suggests a uniform pressure $p_{0}$ and density $\rho_{0}$ in the fluid at rest. Then, the first order in $\epsilon$ describes the acoustic field:

$$
\begin{aligned}
\partial_{t} \tilde{\rho}+\rho_{0} \partial_{i} \tilde{v}_{i} & =0, \\
\rho_{0} \partial_{t} \tilde{v}_{i} & =-\partial_{i} \tilde{p} .
\end{aligned}
$$

Taking the divergence of Eq. (8b) and using the equation of state [Eq. (3)] up to the first order in $\epsilon$ yields the
d'Alembert equation:

$$
\partial_{t t}^{2} \tilde{p}-c_{0}^{2} \partial_{i i p}^{2} \tilde{p}=0
$$

The second order in $\epsilon$ unveils the main nonlinear effects:

$$
\begin{aligned}
& \partial_{t} \bar{\rho}+\partial_{i} \tilde{\rho} \tilde{v}_{i}+\rho_{0} \partial_{i} \bar{v}_{i}=0, \\
& \partial_{t} \rho_{0} \bar{v}_{i}+\partial_{t} \tilde{\rho} \tilde{v}_{i}+\rho_{0} \partial_{j} \tilde{v}_{i} \tilde{v}_{j}=-\partial_{i} \bar{p} \\
& \bar{p}=c_{0}{ }^{2} \bar{\rho}+\frac{1}{2} \Gamma \tilde{\rho}^{2}
\end{aligned}
$$

To facilitate comparison with earlier works $[5,12,13]$, we consider the average of the quantities of interest, instead of the time integral as suggested by the physical setting: $\langle x\rangle=(1 / \tau) \int_{-\tau / 2}^{\tau / 2} x(t) d t$. Due to the finite duration $\tau$ of the pulse, any first-order quantity $\tilde{x}$ satisfies

$$
\left\langle\partial_{t} \tilde{x}\right\rangle=\tilde{x}(\tau / 2)-\tilde{x}(-\tau / 2)=0 .
$$

This allows us to simplify Eqs. (10a)-(10c):

$$
\begin{aligned}
\partial_{i}\left\langle\tilde{\rho} \tilde{v}_{i}\right\rangle+\rho_{0} \partial_{i}\left\langle\bar{v}_{i}\right\rangle & =0, \\
\rho_{0}\left\langle\partial_{j} \tilde{v}_{i} \tilde{v}_{j}\right\rangle & =-\partial_{i}\langle\bar{p}\rangle, \\
\bar{p} & =c_{0}{ }^{2} \bar{\rho}+\frac{1}{2} \Gamma\langle\tilde{\rho}\rangle^{2} .
\end{aligned}
$$

Substituting Eqs. (8a) and (8b) into $\rho_{0}\left\langle\partial_{j} \tilde{v}_{i} \tilde{v}_{j}\right\rangle$ and then using integration by part, we get

$$
\rho_{0}\left\langle\partial_{j} \tilde{v}_{i} \tilde{v}_{j}\right\rangle=\partial_{i}\langle\mathcal{L}\rangle,
$$

with $\mathcal{L}=\mathcal{K}-\mathcal{V}$ the Lagrangian density of the wave, with the acoustic kinetic energy $\mathcal{K}=(1 / 2) \rho_{0} \tilde{v}_{j} \tilde{v}_{j}$ and the acoustic potential energy $\mathcal{V}=\left(\tilde{p}^{2} / 2 \rho_{0} c_{0}^{2}\right)$.

\section{Lagrangian pressure and Brillouin tensor}

In order for the fluid to be at mechanical equilibrium, one must satisfy Eq. (12b). Substituting Eq. (13) in Eq. (12b) yields the Lagrangian pressure:

$$
\langle\bar{p}\rangle=\mathcal{C}-\langle\mathcal{L}\rangle
$$

with $\mathcal{C}$ a constant independent of the position in the fluid [39]. Similarly to the monofrequency case $[32,39]$, a consequence of Eq. (14) is to set the value of the density $\bar{\rho}$ to fulfill Eq. (12c) so that the nonlinearities in the equation of state play no role in the average acoustic radiation pressure of acoustic pulses. We note that this assertion is valid only over the whole duration of the pulse, while $\Gamma$ has a major influence on the detailed dynamics [30]. 
Taking the time average of Eq. (5) and expanding up to the second order in $\epsilon$, we get

$$
\oint_{\partial \Omega}\left\langle\mathcal{B}_{i j}\right\rangle d S_{j}=-\mathcal{F}_{i}
$$

with $\left\langle\mathcal{B}_{i j}\right\rangle=\left\langle\rho_{0} \tilde{v}_{i} \tilde{v}_{j}-\mathcal{L} \delta_{i j}\right\rangle$ the Brillouin tensor, which remains the same as in the monofrequency regime. Equation (15) is the starting point to compute the acoustic radiation force on arbitrarily shaped objects [40] without additional restrictions of size other than satisfying the finite-duration conditions detailed previously.

\section{Acoustic radiation force on small spheres}

It is worth noting that, had Eq. (13) been valid everywhere inside $\Omega$, Eq. (15) would vanish. This suggests to decompose the acoustic field quantities $\tilde{x}$ into a background incident acoustic field, denoted by the subscript $\tilde{x}_{\text {in }}$, [that fulfills (13) and therefore generates no force]; and a scattered acoustic field due to the particle, denoted by the subscript $\tilde{x}_{\mathrm{sc}}$ :

$$
\begin{aligned}
& \tilde{\rho}=\tilde{\rho}_{\text {in }}+\tilde{\rho}_{\mathrm{sc}}, \\
& \tilde{p}=\tilde{p}_{\text {in }}+\tilde{p}_{\mathrm{sc}}, \\
& \tilde{v}_{i}=\tilde{v}_{i, \mathrm{in}}+\tilde{v}_{i, \mathrm{sc}}, \\
& \tilde{\phi}=\tilde{\phi}_{\text {in }}+\tilde{\phi}_{\mathrm{sc}},
\end{aligned}
$$

where we introduce the convenient potential $\tilde{\phi}$, with $\tilde{v}_{i}=$ $\partial_{i} \tilde{\phi}$ and $\tilde{p}=-\rho_{0} \partial_{t} \tilde{\phi}$.

In order to evaluate the scattered field, it is convenient to work in the frequency domain. Any real time-varying functions $\tilde{x}$ can be decomposed into its monofrequency components $\hat{x}(\omega) e^{-i \omega t}$ :

$$
\tilde{x}=\frac{1}{2} \int_{0}^{\infty}\left(\hat{x} e^{-i \omega t}+\hat{x}^{*} e^{i \omega t}\right) d \omega .
$$

So far, we assume only that either the particle is nonresonant or that (i) the acoustic excitation has some periodicity or (ii) the time separation between pulses is long enough to let the scattered field radiated by the particle decay to negligible levels. We now assume that the particle radius $a$ is much smaller than the acoustic wavelength, which yields relatively simple expressions for the scattered field measured at a distance $r \gg \lambda[5,12,13]$ :

$$
\begin{aligned}
\hat{\phi}_{\mathrm{sc}} e^{-i \omega t}= & -f_{1}(\omega) \frac{a^{3}}{3 \rho_{0}} \frac{\left.\partial_{t} \hat{\rho}_{\mathrm{in}} e^{-i \omega t}\right|_{p}}{r} \\
& -f_{2}(\omega) \frac{a^{3}}{2} \partial_{i}\left[\frac{\left.\hat{v}_{i, \mathrm{in}} e^{-i \omega t}\right|_{p}}{r}\right],
\end{aligned}
$$

where $\left.x\right|_{p}$ indicates that $x$ is evaluated at the location of the particle (0) at the retarded time $\left(t-r / c_{0}\right)$. We note that these functions still depend on space due to the time-retarded argument. The monopole and dipole scattering coefficients $f_{1}$ and $f_{2}$ are complex numbers, which depend on the chosen convention $\hat{x}(\omega) e^{-i \omega t}$. Their value can be found in the comprehensive study by Karlsen and Bruus [13].

Substituting Eqs. (16) in Eq. (15), and neglecting squares of $\tilde{\phi}_{\text {in }}$ as they model a wave without any interaction with a particle, therefore yielding no momentum exchange, and the squares of $\tilde{\phi}_{\text {sc }}$ proportional to $a^{6}$ and therefore negligible for a small particle, we get

$$
\begin{aligned}
\mathcal{F}_{i}= & -\int_{\partial \Omega_{f}}\left(\left\langle\frac{\tilde{p}_{\mathrm{in}} \tilde{p}_{\mathrm{sc}}}{\rho_{0} c_{0}^{2}}\right\rangle-\left\langle\rho_{0} \tilde{v}_{k, \text { in }} \tilde{v}_{k, \mathrm{sc}}\right\rangle\right) \delta_{i}^{j} \\
& +\rho_{0}\left\langle\tilde{v}_{i, \text { in }} \tilde{v}_{j, \mathrm{sc}}\right\rangle+\rho_{0}\left\langle\tilde{v}_{i, \mathrm{sc}} \tilde{v}_{j, \text { in }}\right\rangle d S_{j} .
\end{aligned}
$$

Noting that this integral involves only the boundary of the system, we consider a mathematically equivalent system with no particle but with a monopole and dipole source as given in Eq. (18). Using the Gauss theorem, this surface integral can now be replaced by a volume integral over the closed virtual domain $\Omega^{\dagger}$ that encompasses $\Omega$ and the previous location of the particle. After some calculus (see Ref. [12] for a detailed derivation), we get

$$
\mathcal{F}_{i}=-\int_{\Omega^{\dagger}} \rho_{0}\left\langle\tilde{v}_{i, \text { in }}\left(\partial_{j j}^{2}-\frac{1}{c_{0}^{2}} \partial_{t t}^{2}\right) \tilde{\phi}_{\mathrm{sc}}\right\rangle d V .
$$

Similarly to the previous studies $[5,12,13]$, we recall that the d'Alembert equation acting on the monopole and dipole terms, Eq. (9), result in a singular density point $\left.\hat{\rho}_{\text {in }} e^{-i \omega t}\right|_{p} \delta(\mathbf{r})$ and a singular velocity point $\left.\hat{v}_{i, \text { in }} e^{-i \omega t}\right|_{p} \delta(\mathbf{r})$, with $\delta$ the Dirac distribution.

$$
\begin{aligned}
\left(\partial_{j j}^{2}-\frac{1}{c_{0}^{2}} \partial_{t t}^{2}\right) \hat{\phi}_{\mathrm{sc}} e^{-i \omega t}= & \left.f_{1}(\omega) \frac{4 \pi a^{3}}{3 \rho_{0}} \partial_{t} \hat{\rho}_{\mathrm{in}} e^{-i \omega t}\right|_{p} \delta(\mathbf{r}) \\
& +\left.f_{2}(\omega) 2 \pi a^{3} \partial_{j} \hat{v}_{j, \mathrm{in}} e^{-i \omega t}\right|_{p} \delta(\mathbf{r})
\end{aligned}
$$

Integrating Eq. (21) over all angular frequencies $\omega$, we get the time-dependent scattered field:

$$
\begin{aligned}
& \left(\partial_{j j}^{2}-\frac{1}{c_{0}^{2}} \partial_{t t}^{2}\right) \tilde{\phi}_{\mathrm{sc}} \\
& \quad=\frac{4 \pi a^{3}}{3}\left[\left.\frac{1}{\rho_{0} c_{0}^{2}} \partial_{t} \delta(\mathbf{r}) \tilde{p}_{m}\right|_{p}+\left.\partial_{j} \delta(\mathbf{r}) \tilde{v}_{j, d}\right|_{p}\right]
\end{aligned}
$$

with the monopole and dipole scattered fields:

$$
\left.\tilde{p}_{m}\right|_{p}=\left.\frac{1}{2} \int_{0}^{\infty} f_{1}(\omega) \hat{p}_{\text {in }} e^{-i \omega t}\right|_{p}+\left.f_{1}^{*}(\omega) \hat{p}_{\text {in }}^{*} e^{i \omega t}\right|_{p} d \omega
$$




$$
\left.\tilde{v}_{j, d}\right|_{p}=\left.\frac{3}{4} \int_{0}^{\infty} f_{2}(\omega) \hat{v}_{j, \text { in }} e^{-i \omega t}\right|_{p}+\left.f_{2}^{*}(\omega) \hat{v}_{j, \text { in }}^{*} e^{i \omega t}\right|_{p} d \omega,
$$

where the $\partial_{t}, \partial_{j}$ operators and the $\delta$ distribution commute with the integral over angular frequencies.

Substituting Eq. (22) in Eq. (20) and using the Gauss theorem (see Ref. [12] for additional details), the timeretarded argument in the scattered fields is simplified by the Dirac function, so that Eqs. (23) and (24) can now be evaluated at the location of the particle. We obtain a first expression of the acoustic radiation force:

$$
\mathcal{F}_{i}=-\frac{4 \pi a^{3}}{3}\left[\left\langle\tilde{v}_{i, \text { in }} \frac{1}{c_{0}^{2}} \partial_{t} \tilde{p}_{m}\right\rangle-\left\langle\rho_{0} \tilde{v}_{j, d} \partial_{j} \tilde{v}_{i, \text { in }}\right\rangle\right] .
$$

Integrating Eq. (25) by part then yields

$$
\mathcal{F}=-\frac{4 \pi a^{3}}{3}\left[\left\langle\frac{1}{\rho_{0} c_{0}^{2}} \tilde{p}_{m} \nabla \tilde{p}_{\text {in }}\right\rangle-\left\langle\rho_{0} \tilde{\mathbf{v}}_{d} \cdot \nabla \tilde{\mathbf{v}}_{\text {in }}\right\rangle\right],
$$

which in the monofrequency regime simplifies into the Gor'kov equation $[5,12,13]$.

\section{RESULTS AND DISCUSSION}

In the following, we consider the effect of pulses on the motion of particles. While Eq. (26) is valid for any finite-duration pulse, the general calculation is complicated by the convolution products in Eqs. (23) and (24). These equations can be simplified (i) by considering narrowbandwidth acoustic beams so the variation of all quantities but the wave spectrum can be neglected over the integration bandwidth, or (ii) by considering inviscid fluids where the scattering coefficients can be factored out of the integrals.

When a particular example is needed, we use Gaussian plane-wave packets propagating along the $x$ direction $\tilde{p}_{\text {in }}(\theta)=p_{\max } w(\theta)$ with

$$
w(\theta)=\exp \left(-\sigma \theta^{2}\right) \cos (\omega \theta)
$$

with $\theta=t-\mathbf{s} \cdot \mathbf{r}$ the retarded time of the wave, where $\mathbf{s}=$ $\left(1 / c_{0}\right) \mathbf{e}_{x}$. Advantageously, calculations with these waves are relatively straightforward but still allow tuning the pulse width (chosen as $\Theta=\sqrt{2 / \sigma}$ to account for at least $99.5 \%$ of the pulse energy) by changing $\sigma$. For such traveling plane waves, the velocity field is given by $\tilde{v}_{\text {in }}=$ $\tilde{p}_{\text {in }}\left(1 / \rho_{0} c_{0}\right) \mathbf{e}_{x}$. A helpful quantity when studying the acoustic radiation force is the mean energy density $\langle\mathcal{E}\rangle$, defined as the total energy flux per unit length $\langle\mathcal{E}\rangle=\mathbf{s} \cdot\langle\tilde{p} \tilde{\mathbf{v}}\rangle$. In the case of a Gaussian wave packet, it reads

$$
\begin{aligned}
\langle\mathcal{E}\rangle & =\left\langle\mathcal{E}_{\infty}\right\rangle\left(1+e^{-\omega^{2} / 2 \sigma}\right), \\
\text { with }\left\langle\mathcal{E}_{\infty}\right\rangle & =\frac{\tilde{p}_{\max }^{2}}{\rho_{0} c_{0}^{2}} \frac{1}{\tau} \sqrt{\frac{\pi}{8 \sigma}} .
\end{aligned}
$$

The time and frequency spread of the wave packet (defined as the standard deviation in time and frequency space) will also be useful, and read (respectively)

$$
\begin{gathered}
\delta t=\frac{1}{\sqrt{2 \sigma}}, \\
\delta f=\sqrt{\frac{\sigma}{2 \pi^{2}}} .
\end{gathered}
$$

\section{A. Narrow-band waves in a thermoviscous fluid}

We first consider an acoustic wave packet with a bandwidth $\delta f=\delta \omega / 2 \pi$ narrow enough to neglect the variations of $f_{1}$ and $f_{2}$ over this frequency band. Before discussing the force, we note that this assumption poses some constraints on the pulse duration. According to Settnes, Karlsen, and Bruus, this requires that the thermoviscous boundary layer thickness $\Lambda_{t v}$ to particle radius ratio do not change much over $\delta \omega[12,13]$, which yields $\delta \omega \ll$ $2 \omega\left(a / \Lambda_{t v}\right)$, with $\Lambda_{t v}=\min \left(\Lambda_{\text {visc }}, \Lambda_{\text {therm }}\right)$. According to the Gabor limit, $\delta t \delta f \geq \frac{1}{2 \pi}$ [41], with the equality being true when using Gaussian wave packets. Combining these inequalities with Eq. (29a) yields a lower limit for the number of periods $n_{T}$ of the pulse:

$$
n_{T} \simeq \sqrt{\frac{2 \omega^{2}}{\sigma}} \gg \frac{\Lambda_{t v}}{a} .
$$

While this inequality does not contradict Eq. (1), their combination restricts this thermoviscous case to lowamplitude long-duration wave packets.

Separating the real and imaginary parts of $f_{1}=f_{1}{ }^{r}+i f_{1}{ }^{i}$ and $f_{2}=f_{2}^{r}+i f_{2}^{i}$ and noting that in the current complex convention for the scattering coefficients we have $i\left(\hat{x} e^{-i \omega t}-\hat{x}^{*} e^{i \omega t}\right)=-\frac{1}{\omega} \partial_{t}\left(\hat{x} e^{-i \omega t}+\hat{x}^{*} e^{i \omega t}\right)$, yields

$$
\begin{aligned}
\left.\tilde{p}_{m}\right|_{p} & =\left.f_{1}^{r} \tilde{p}_{\text {in }}\right|_{p}-\left.\frac{f_{1}^{i}}{\omega} \partial_{t} \tilde{p}_{\text {in }}\right|_{p}, \\
\left.\tilde{v}_{j, d}\right|_{p} & =\frac{3}{2}\left(\left.f_{2}^{r} \tilde{v}_{j, \text { in }}\right|_{p}-\left.\frac{f_{2}{ }^{i}}{\omega} \partial_{t} \tilde{v}_{j, \text { in }}\right|_{p}\right),
\end{aligned}
$$

where, consistently with the narrow-band hypothesis, the variations of $1 / \omega$ over the frequency interval are neglected.

Substituting Eqs. (31) and (32) in Eq. (26), and omitting the real part of $f_{1}$ and $f_{2}$ that do not contribute to the acoustic radiation force for traveling waves up to $O\left(a^{6}\right)$ [42], we 
get the force due to a narrow-band traveling wave $\tilde{p}(\theta)$ in a thermoviscous fluid:

$$
\mathcal{F}=-\mathbf{s} \frac{4 \pi a^{3}}{3} \frac{1}{\omega \rho_{0} c_{0}^{2}}\left[f_{1}^{i}-\frac{3 f_{2}^{i}}{2}\right]\left\langle\left(\partial_{\theta} \tilde{p}_{\text {in }}\right)^{2}\right\rangle
$$

which agrees with Ref. [12] except for a minor typo in the sign of $f_{1}^{i}$.

In the case of Gaussian wave packets, the force simplifies into

$$
\mathcal{F}=-\mathbf{s} \omega \frac{4 \pi a^{3}}{3}\left[f_{1}^{i}-\frac{3 f_{2}{ }^{i}}{2}\right]\left\langle\mathcal{E}_{\infty}\right\rangle
$$

Careful inspection of Eq. (34) reveals that the acoustic radiation force decreases in $1 / \tau$. This mathematical artifact is due to the time averaging of the force over the time $\tau$ chosen arbitrarily. Hence, a better measure of the effect of the acoustic radiation force of finite-duration pulses is the transmitted acoustic radiation momentum $\mathbf{q}=\tau \mathcal{F}$, which depends on the physically relevant pulse width $(\Theta=\sqrt{2 / \sigma})$ but is independent of the pulse duration.

\section{B. Arbitrary waves in an inviscid fluid}

When the particle is much larger than the viscoacoustic or thermoacoustic boundary layers, the scattering coefficients $f_{1}(\omega)=f_{1}^{r}$ and $f_{2}(\omega)=f_{2}^{r}$ are purely real and independent of the frequency [5], and therefore can be factored out of the integrals in Eqs. (23) and (24), which yields

$$
\mathcal{F}=-\frac{4 \pi a^{3}}{3} \nabla\langle\mathcal{U}\rangle
$$

with the dynamic Gor'kov potential:

$$
\mathcal{U}=\frac{f_{1}}{2 \rho_{0} c_{0}^{2}} \tilde{p}_{\text {in }}^{2}-\frac{3 \rho_{0} f_{2}}{4} \tilde{\mathbf{v}}_{\text {in }}^{2}
$$

\section{Traveling waves}

In the case of traveling waves, $\tilde{p}_{\text {in }}$ and $\tilde{v}_{i, \text { in }}$ are only functions of $\theta=t-\mathbf{s} \cdot \mathbf{r}$ with $\mathbf{s}$ the wave slowness. Therefore, $\mathcal{U}$ is a function of $\theta$ only, such that

$$
\nabla\langle\mathcal{U}\rangle=\left\langle\partial_{\theta} \mathcal{U} \nabla \theta\right\rangle=-\mathbf{s}\left\langle\partial_{t} \mathcal{U}\right\rangle=O\left(a^{6}\right),
$$

which vanishes up to the small terms in $O\left(a^{6}\right)$ that are neglected in Eq. (20). Extrapolating from the monofrequency regime, one may reasonably expect that higherorder terms called scattering force will dominate the acoustic radiation force $[8,23]$ in this case.

\section{Interference of two plane-wave packets}

We next consider two interfering plane-wave packets $\tilde{p}=\tilde{p}\left(\theta^{+}\right)+\tilde{p}\left(\theta^{-}\right)$, as illustrated in Fig. 1(c). For the sake of generality, the two packets intersect with an angle $2 \eta_{R}$, so that $\theta^{+}=t+s_{x} x-s_{z} z$ and $\theta^{-}=t-s_{x} x-s_{z} z$, with $s_{x}=\sin \eta_{R} / c_{0}$ and $s_{z}=\cos \eta_{R} / c_{0}$. While $\eta_{R}=\pi / 2$ is relevant for a frontal interference $[43,44]$, the general case applies to surface-acoustic-wave-based tweezers such as the ones proposed by Collins et al., where $\eta_{R}$ plays the role of the Rayleigh angle that the acoustic radiation makes with the substrate [45].

Assuming that the incident wave reads $\tilde{p}_{\text {in }}=p_{\max }$ $\left[\tilde{w}\left(\theta^{+}\right)+\tilde{w}\left(\theta^{-}\right)\right]$, with $w$ given in Eq. (27), we have $\tilde{v}_{\text {in }, x}=-\left(p_{\max } s_{x} / \rho_{0} c_{0}\right)\left[\tilde{w}\left(\theta^{+}\right)-\tilde{w}\left(\theta^{-}\right)\right]$and $\tilde{v}_{\text {in }, z}=\tilde{p}_{\text {in }} s_{z} /$ $\rho_{0}$. This yields the Gor'kov potential and the acoustic radiation momentum $\mathbf{q}$ transferred by an acoustic wave during the interference of two wavelets:

$$
\begin{aligned}
\mathcal{U} & =\left\langle\mathcal{E}_{\infty}\right\rangle \Psi\left[\cos \left(2 \omega s_{x} x\right)+e^{-\omega^{2} / 2 \sigma}\right] G\left(s_{x} x\right), \\
\mathbf{q} & =-2 \omega s_{x} \frac{4 \pi a^{3}}{3} \tau \Psi\left\langle\mathcal{E}_{\infty}\right\rangle \zeta\left(s_{x} x\right) \mathbf{e}_{x}, \\
\Psi & =f_{1}^{r}-\cos \left(2 \eta_{R}\right) \frac{3}{2} f_{2}^{r},
\end{aligned}
$$

with $G\left(s_{x} x\right)=e^{-2 \sigma\left(s_{x} x\right)^{2}}$. The acoustic contrast factor is identical to the monofrequency case [46]. The dimensionless restoring force $\zeta$ reads

$$
\begin{aligned}
\zeta\left(s_{x} x\right)= & 2\left[\frac{2 \sigma s_{x} x}{\omega}\left[e^{-\omega^{2} / 2 \sigma}+\cos \left(2 \omega s_{x} x\right)\right]+\sin \left(2 \omega s_{x} x\right)\right] \\
& \times G\left(s_{x} x\right) .
\end{aligned}
$$

Examples of the Gor'kov potential for various values of $\sigma / \omega^{2}$ are shown in Fig. 2. For acoustic tweezers applications, it is often desirable to trap a single particle and not its neighbors. Such selective capture requires reducing the spatial extent of the trapping region. Here, this can be conveniently achieved by decreasing the pulse width parameter $\sigma$. We note that this time-dependent strategy comes on top of existing solutions such as acoustic vortices $[20,21,23,47]$, and the two methods can therefore reinforce each other.

Although using increasingly narrow pulses reduces the spatial extent of the wave, it does not necessarily improve its selectivity. Indeed, the acoustic radiation force changes sign depending on the particles' contrast factor $[12,13]$. Cells and particles have a positive contrast factor, meaning that these particles are trapped at the local minima of $\langle\mathcal{U}\rangle / \Psi\left\langle\mathcal{E}_{\infty}\right\rangle$. However, the energy landscape shown in Fig. 2 has multiple local minima, which may result in as many trapping positions. A better measure of selectivity $\Delta \zeta_{\max }$ is then how strongly the particles are bond to their local trap, especially the difference of restoring force 


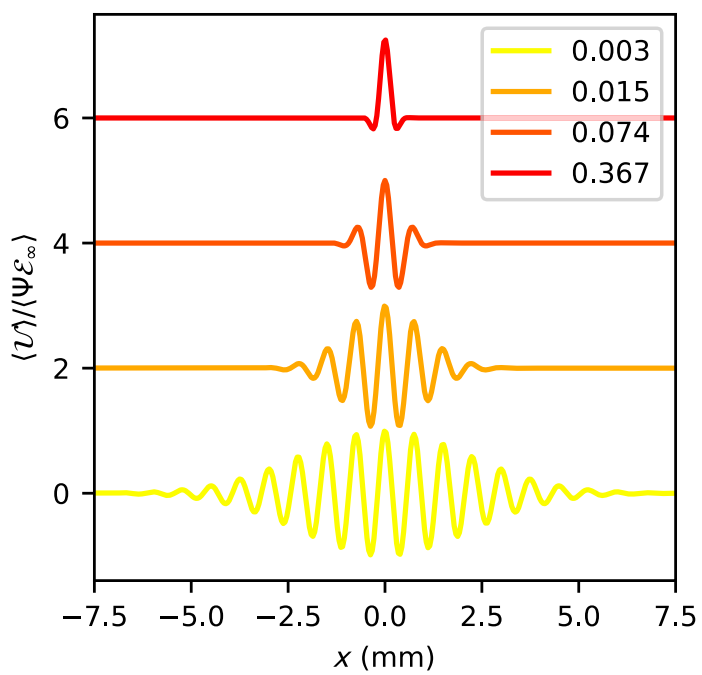

FIG. 2. Normalized Gor'kov potential $\langle\mathcal{U}\rangle /\left\langle\Psi \mathcal{E}_{\infty}\right\rangle$ from the interference of two wave packets for different values of $\sigma / \omega^{2}$ in water (acoustic frequency $1 \mathrm{MHz}$, sound speed $c_{0}=1500 \mathrm{~m} / \mathrm{s}$ ). The vertical offset between curves is added to improve clarity.

between the strongest trap (primary trap) and the second strongest one (secondary trap) [48]. The variation of selectivity depending of the pulse width $\sigma$ is shown in Fig. 3. Within the limits of the theory (particle much smaller than the highest frequency in the spectrum, which ultimately increases with $\sqrt{\sigma}$ ), the trapping selectivity of negative $\Psi$ particles always increases for narrower traps. However, positive-contrast particles have an optimum selectivity for $\sigma=0.033 \omega^{2}$.
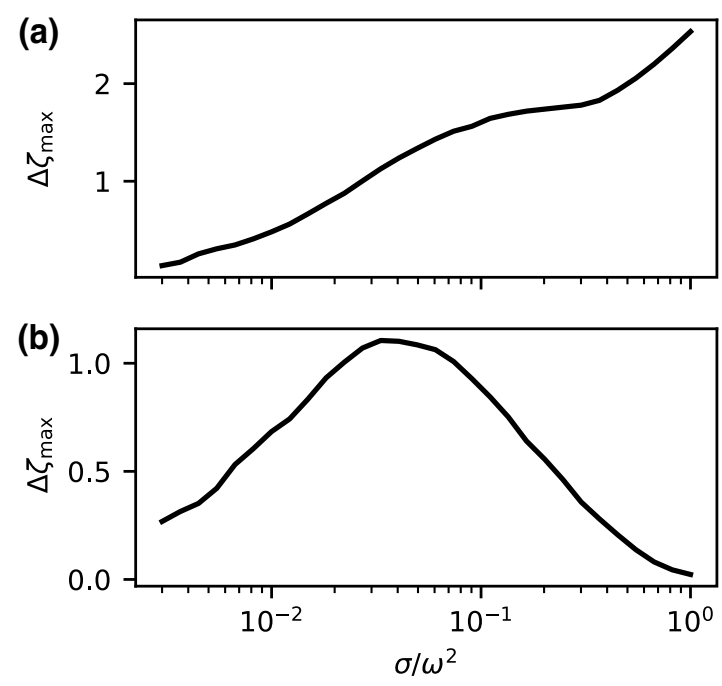

FIG. 3. Selectivity of the acoustic radiation force obtained during the interference of two wavepackets of various dimensionless widths $\sigma / \omega^{2}$. (a) Negative acoustic contrast $\Psi$. (b) Positive acoustic contrast $\Psi$. The selectivity $\Delta \zeta_{\max }$ measures the difference of restoring force between primary and secondary traps.
In order to compare this value, obtained for a pair of Gaussian wave packets, to the value used by Collins et al. in their experimental study [19] with a triangular wave, we use a least-squares fitting to find the best approximation for $\sigma$. For a triangular wave packet with $n_{T}$ periods, we get $\sigma / \omega^{2} \simeq 10.041 / 2 \pi n_{T}^{2}$. For the 10-period wave packet used in their experiments, we get $\sigma \simeq 0.016 \omega^{2}$. According to Fig. 3, this suggests that reducing the pulse duration twofold would not only yield a more localized trap but would also double the difference of force between primary and secondary traps. This is a very strong incentive to develop acoustofluidic devices in the pulsed regime using broadband acoustic transducers.

\section{CONCLUSION}

Acoustic radiation force has long been considered a steady-state time-averaged phenomenon. Here, we derive an expression of the acoustic radiation force acting on spheres for arbitrary acoustic pulses, as long as the pulse bandwidth satisfies (i) the small sphere condition $f_{\max } \ll$ $\frac{c_{0}}{a}$ and (ii) pulse durations are short enough to neglect the sphere displacement compared to the wavelength $f_{\min } \simeq$ $1 / \tau \gg f_{\max } \epsilon^{2}$. We provide an extension of the Gor'kov formula in the case of wave packets and other unsteady acoustic fields. Even in the time domain, traveling waves in an inviscid fluid do not generate a net force up to $a^{6}$, while standing waves do yield a force proportional to $a^{3}$. Based on the complex monopole and dipole-scattering coefficients, our model can account for thermoviscous effects that can considerably enhance the acoustic radiation force of traveling waves. This model provides a theoretical foundation for the use of pulsed acoustic waves to enhance acoustic tweezers selectivity, and clarifies that an acoustic radiation force exists even for a single acoustic period.

\section{ACKNOWLEDGMENTS}

This work is supported by the National Natural Science Foundation of China with Grants No. 51950410582, No. 61874033, and No. 61674043, the Science Foundation of Shanghai Municipal Government with Grant No. 18ZR1402600, the State Key Lab of ASIC and System, Fudan University with Grants No. 2018MS003 and No. 2020KF006, ISITE-ULNE (ERC Generator program and Talent project) and Institut Universitaire de France.

[1] L. Rayleigh, On the pressure of vibrations, Phil. Mag. 3, 338 (1902).

[2] L. Rayleigh, On the momentum and pressure of gaseous vibrations, and on the connexion with the virial theorem, Phil. Mag. 10, 364 (1905). 
[3] L. V. King, On the acoustic radiation pressure on spheres, Proc. R. Soc. London. Ser. Math. Phys. Sci. 147, 212 (1934).

[4] K. Yosioka and Y. Kawasima, Acoustic radiation pressure on a compressible sphere, Acta Acust. United Acust. 5, 167 (1955).

[5] L. P. Gor'kov, On the forces acting on a small particle in an acoustical field in an ideal fluid, Sov. Phys. Dokl. 6, 773 (1962).

[6] D. Baresch, J.-L. Thomas, and R. Marchiano, Threedimensional acoustic radiation force on an arbitrarily located elastic sphere, J. Acoust. Soc. Am. 133, 25 (2013).

[7] G. T. Silva, J. H. Lopes, J. P. Leãao-Neto, M. K. Nichols, and B. W. Drinkwater, Particle Patterning by Ultrasonic Standing Waves in a Rectangular Cavity, Phys. Rev. Appl. 11, 054044 (2019).

[8] O. A. Sapozhnikov and M. R. Bailey, Radiation force of an arbitrary acoustic beam on an elastic sphere in a fluid, J. Acoust. Soc. Am. 133, 661 (2013).

[9] A. A. Doinikov, Acoustic radiation force on a spherical particle in a viscous heat-conducting fluid. I. General formula, J. Acoust. Soc. Am. 101, 713 (1997).

[10] A. A. Doinikov, Acoustic radiation force on a spherical particle in a viscous heat-conducting fluid. II. Force on a rigid sphere, J. Acoust. Soc. Am. 101, 722 (1997).

[11] A. A. Doinikov, Acoustic radiation force on a spherical particle in a viscous heat-conducting fluid. III. Force on a liquid drop, J. Acoust. Soc. Am. 101, 731 (1997).

[12] M. Settnes and H. Bruus, Forces acting on a small particle in an acoustical field in a viscous fluid, Phys. Rev. E 85, 016327 (2012).

[13] J. T. Karlsen and H. Bruus, Forces acting on a small particle in an acoustical field in a thermoviscous fluid, Phys. Rev. E 92, 043010 (2015).

[14] G. T. Silva, S. Chen, J. F. Greenleaf, and M. Fatemi, Dynamic ultrasound radiation force in fluids, Phys. Rev. E 71, 056617 (2005).

[15] G. T. Silva, M. W. Urban, and M. Fatemi, Multifrequency radiation force of acoustic waves in fluids, Phys. D: Nonlinear Phenom. 232, 48 (2007).

[16] X. Ding, S.-C. S. Lin, B. Kiraly, H. Yue, S. Li, I.-K. Chiang, J. Shi, S. J. Benkovic, and T. J. Huang, On-chip manipulation of single microparticles, cells, and organisms using surface acoustic waves, Proc. Natl. Acad. Sci. 109, 11105 (2012).

[17] Z. Tian, S. Yang, P.-H. Huang, Z. Wang, P. Zhang, Y. Gu, H. Bachman, C. Chen, M. Wu, Y. Xie, et al., Wave number-spiral acoustic tweezers for dynamic and reconfigurable manipulation of particles and cells, Sci. Adv. 5, eaau6062 (2019).

[18] P. Kang, Z. Tian, S. Yang, W. Yu, H. Zhu, H. Bachman, S. Zhao, P. Zhang, Z. Wang, R. Zhong, et al., Acoustic tweezers based on circular, slanted-finger interdigital transducers for dynamic manipulation of micro-objects, Lab. Chip 20, 987 (2020).

[19] D. J. Collins, C. Devendran, Z. Ma, J. W. Ng, A. Neild, and Y. Ai, Acoustic tweezers via sub-time-of-flight regime surface acoustic waves, Sci. Adv. 2, e1600089 (2016).

[20] A. Riaud, M. Baudoin, O. Bou Matar, L. Becerra, and J.-L. Thomas, Selective Manipulation of Microscopic Particles with Precursor Swirling Rayleigh Waves, Phys. Rev. Appl. 7, 024007 (2017).

[21] M. Baudoin, J.-C. Gerbedoen, A. Riaud, O. Bou Matar, N. Smagin, and J.-L. Thomas, Folding a focalized acoustical vortex on a flat holographic transducer: Miniaturized selective acoustical tweezers, Sci. Adv. 5, eaav1967 (2019).

[22] C. R. Courtney, B. W. Drinkwater, C. E. Demore, S. Cochran, A. Grinenko, and P. D. Wilcox, Dexterous manipulation of microparticles using bessel-function acoustic pressure fields, Appl. Phys. Lett. 102, 123508 (2013).

[23] D. Baresch, J.-L. Thomas, and R. Marchiano, Observation of a Single-Beam Gradient Force Acoustical Trap for Elastic Particles: Acoustical Tweezers, Phys. Rev. Lett. 116, 024301 (2016).

[24] A. Riaud, J.-L. Thomas, E. Charron, A. Bussonnière, O. Bou Matar, and M. Baudoin, Anisotropic Swirling Surface Acoustic Waves from Inverse Filtering for OnChip Generation of Acoustic Vortices, Phys. Rev. Appl. 4, 034004 (2015).

[25] V. Zharov, T. Malinsky, and R. Kurten, Photoacoustic tweezers with a pulsed laser: Theory and experiments, J. Phys. D: Appl. Phys. 38, 2662 (2005).

[26] T. Pezeril, G. Saini, D. Veysset, S. Kooi, P. Fidkowski, R. Radovitzky, and K. A. Nelson, Direct Visualization of Laser-Driven Focusing Shock Waves, Phys. Rev. Lett. 106, 214503 (2011).

[27] A. Longhorn, The unsteady, subsonic motion of a sphere in a compressible inviscid fluid, Q. J. Mech. Appl. Math. 5, 64 (1952).

[28] M. Parmar, A. Haselbacher, and S. Balachandar, Generalized Basset-Boussinesq-Oseen Equation for Unsteady Forces on a Sphere in a Compressible Flow, Phys. Rev. Lett. 106, 084501 (2011).

[29] S. Annamalai and S. Balachandar, Faxén form of timedomain force on a sphere in unsteady spatially varying viscous compressible flows, J. Fluid Mech. 816, 381 (2017).

[30] G. T. Silva, S. Chen, and L. P. Viana, Parametric Amplification of the Dynamic Radiation Force of Acoustic Waves in Fluids, Phys. Rev. Lett. 96, 234301 (2006).

[31] S. Chen, G. T. Silva, R. R. Kinnick, J. F. Greenleaf, and M. Fatemi, Measurement of dynamic and static radiation force on a sphere, Phys. Rev. E 71, 056618 (2005).

[32] M. Baudoin and J.-L. Thomas, Acoustic tweezers for particle and fluid micromanipulation, Annu. Rev. Fluid Mech. 52, 205 (2020).

[33] A. Riaud, M. Baudoin, O. Bou Matar, J. Thomas, and P. Brunet, On the influence of viscosity and caustics on acoustic streaming in sessile droplets: An experimental and a numerical study with a cost-effective method, J. Fluid Mech. 821, 384 (2017).

[34] C. Eckart, Vortices and streams caused by sound waves, Phys. Rev. 73, 68 (1948).

[35] L. Rayleigh, On the circulation of air observed in kundt's tubes, and on some allied acoustical problems, Philos. Trans. R. Soc. London 175, 1 (1884).

[36] A. Doinikov, Acoustic radiation pressure on a rigid sphere in a viscous fluid, Proc. R. Soc. London. Ser. A: Math. Phys. Sci. 447, 447 (1994). 
[37] T. Baasch, A. Pavlic, and J. Dual, Acoustic radiation force acting on a heavy particle in a standing wave can be dominated by the acoustic microstreaming, Phys. Rev. E 100, 061102 (2019).

[38] P. B. Muller, M. Rossi, A. G. Marin, R. Barnkob, P. Augustsson, T. Laurell, C. J. Kahler, and H. Bruus, Ultrasound-induced acoustophoretic motion of microparticles in three dimensions, Phys. Rev. E 88, 023006 (2013).

[39] T. Hasegawa, T. Kido, T. Iizuka, and C. Matsuoka, A general theory of Rayleigh and Langevin radiation pressures, Acoustical Sci. Technol. 21, 145 (2000).

[40] Z. Gong, P. L. Marston, and W. Li, T-matrix evaluation of three-dimensional acoustic radiation forces on nonspherical objects in bessel beams with arbitrary order and location, Phys. Rev. E 99, 063004 (2019).

[41] This result differs from the well-known Gabor-Heisenberg result $\delta t \delta f \geq \frac{1}{4 \pi}$ obtained for the uncertainty on density of states as opposed to wave functions. In the former case, evaluating the density of states raises the distributions to the power 2 , thereby reducing their spread by half.

[42] See the case of traveling waves in an inviscid fluid for more details.
[43] Z. I. Mandralis and D. L. Feke, Fractionation of suspensions using synchronized ultrasonic and flow fields, AIChE J. 39, 197 (1993).

[44] A. Lenshof, M. Evander, T. Laurell, and J. Nilsson, Acoustofluidics 5: Building microfluidic acoustic resonators, Lab. Chip 12, 684 (2012).

[45] J. Vanneste and O. Bühler, Streaming by leaky surface acoustic waves, Proc. R. Soc. A: Math., Phys. Eng. Sci. 467, 1779 (2011).

[46] G. Simon, M. A. Andrade, J. Reboud, J. Marques-Hueso, M. P. Desmulliez, J. M. Cooper, M. O. Riehle, and A. L. Bernassau, Particle separation by phase modulated surface acoustic waves, Biomicrofluidics 11, 054115 (2017).

[47] Z. Gong and M. Baudoin, Three-Dimensional Trapping and Assembly of Small Particles with Synchronized Spherical Acoustical Vortices, Phys. Rev. Appl. 14, 064002 (2020).

[48] The ratio of acoustic radiation force could also have been considered here, but yields misleading results when the restoring force of the primary and secondary traps becomes vanishingly small. 